\title{
Experimental and numerical investigation of friction-induced vibration of a beam-on-beam in contact with friction
}

\author{
A. Meziane ${ }^{\mathrm{a}, *}$, L. Baillet $^{\mathrm{b}}$, B. Laulagnet $^{\mathrm{c}}$ \\ a Laboratoire de Mécanique des Contacts et des Solides, INSA-Lyon, CNRS UMR5259 20, Avenue Albert Einstein, 69621 Villeurbanne, France \\ ${ }^{\mathrm{b}}$ Laboratoire de Géophysique Interne et Tectonophysique, Université Joseph Fourier, CNRS, BP 53, 38041 Grenoble Cedex 9, France \\ ${ }^{\mathrm{c}}$ Laboratoire de Vibrations et Acoustique, INSA-Lyon 20, Avenue Albert Einstein, 69621 Villeurbanne, France
}

\begin{abstract}
The vibrations generated by friction are responsible for various noises such as squealing, squeaking and chatter. Although these phenomena have been studied for a long time, it is not well-understood. In this study, an experimental and numerical study of friction-induced vibrations of a system composed of two beams in contact is proposed. The experimental system exhibits periodic steady state vibrations of different types. To model and understand this experimental vibratory phenomenon, complex eigenvalue and dynamic transient analyses are performed. In the linear complex eigenvalue analysis, flutter instability occurs via the coalescence of two eigenmodes of the system. This linear study provides an accurate value of the experimental frequency of vibration. To understand what happens physically during frictioninduced instability, a dynamic transient analysis that takes account of the non-linear aspect of a frictional contact is performed. In this analysis, friction-induced instability is characterized by self-sustained vibrations and by stick, slip and separation zones occurring at the surface of the contact. The results stemming from this analysis show that good correlation between numerical and experimental vibrations can be obtained (in time and frequency domains). Moreover, time domain simulations permit understanding the physical phenomena involved in two different vibratory behaviours observed experimentally.
\end{abstract}

\section{Introduction}

When two bodies are in contact with friction, friction-induced vibrations can occur. These vibrations generated by friction are responsible for different noises and stress concentrations. In the case of automobile braking, these vibrations can generate groaning $(<100 \mathrm{~Hz})$, squealing $(1-20 \mathrm{kHz})$, humming and moaning (100$500 \mathrm{~Hz}$ ) and so forth. A wide panorama of vibration dynamics caused by friction has been presented by Ibrahim [1,2], Papinniemi [3], and Kinkaid et al. [4]. Akay [5] has given an overview of friction acoustics. The literature includes many experimental, analytical and numerical studies that show that the friction-induced instability phenomenon is complex and has yet to be brought under full control.

Mills [6] showed that friction-induced instabilities can occur when the friction coefficient decreases with relative velocity. This theory explains that friction-induced vibrations are explained by an instability related to a stick-slip phenomenon. Using the Sprag-Slip model, Spurr [7] highlighted the importance of contact kinematics by obtaining an instability condition on the contact's

\footnotetext{
* Corresponding author. Tel.: +33 4724384 45; fax: +334 78890980 . E-mail address: anissa.meziane@insa-lyon.fr (A. Meziane).
}

angle of incidence with a constant friction coefficient. His model was composed of a rigid articulated bar in friction contact on a rigid surface. In an analytical and experimental study of a commercial braking system, North [8] showed that friction-induced vibrations are due to a coalescence of two eigenfrequencies of the system. Friction-induced instabilities obtained with a constant friction coefficient correspond to self-excited vibrations of the system induced by flutter instability.

Numerical studies relying on the finite element method permits the treatment of a wide range of systems (geometry of the bodies in contact, limit conditions and contact modelling). These numeric studies are mainly based on two types of analysis: complex eigenvalue analysis and dynamic transient analysis. The linear complex eigenvalue analysis permits detection of the stability limit of the system, by analysing its eigenvalues and eigenvectors around the steady sliding state [9-11]. The good agreement with experimental results [12-14] proves that the friction-induced instability domain can be detected with a linear hypothesis in the contact zone. However, the non-linear effects of contact with friction are not negligible when instability occurs. Thus to study the evolution of the vibration of the system during instability, the transient dynamic analysis that takes into account the non-linear aspect of contact with friction is used. This permits obtaining the values of displacements, velocities and accelerations, as well as the values of the 
forces and surfaces of the contact during system vibrations [1517]. More often than not, these numerical analyses are performed separately. Recently, certain numerical works [18-20] have performed both types of analysis and shown that they are complementary.

The difficulties of the phenomenon of friction-induced vibrations stems from the correlation between numerical and experimental results. Most works presenting comparisons between numerical and experimental results succeed in correlating unstable frequencies [12] and sometimes the level of vibration [21], but as far as the authors know the literature provides no examples of numerical studies of the vibrations caused by friction that present correlations between numerical and experimental vibrations in the time domain.

The initial aim of this paper is to show that good correlation (in both time and frequency domains) between numerical and experimental unstable vibrations can be obtained, while the second aim is to demonstrate that the numerical study can explain some of the physical phenomena involved in friction-induced vibrations of the system. Given the complexity of the friction-induced vibration phenomenon, an experimental system has been built up in order to control the number of parameters that play a role in friction-induced vibrations. Firstly, the results obtained in the experimental study of the system are described. Then a numerical study is performed comprising both linear and non-linear analyses followed by the validation of numerical results and a discussion on the physical phenomena involved in friction-induced vibrations.

\section{Experimental study}

\subsection{Experimental rig}

The bench used is shown in Fig. 1. It is composed of three parts:

- a fixed part (I) connected to the ground composed of a frame (9) and a DC-motor (8),

- a part moving in translation (II) composed of the beam $\mathrm{P}^{(2)}(2)$ and its support, that can translate along the $x$-axis in relation to the frame (7) via a screw-nut system (4) driven in rotation by a DC motor (8),

- a part (III) composed of beam $\mathrm{P}^{(1)}(1)$, its support (3), the weight (5) corresponding to the application of a force of $9 \mathrm{~N}$ at point $\mathrm{A}$, and a counterweight (10). The weight of assembly $(3)+(1)$ is counterbalanced by (10) in order to apply the force only with the weight (5). This part free from rotation around the $z$-axis at point $\mathrm{E}$.

Beam $\mathrm{P}^{(1)}$ is clamped at its support at point $\mathrm{A}$ and is in contact with beam $\mathrm{P}^{(2)}$ at point $\mathrm{C}$. As part (III) can rotate at point $\mathrm{E}$, beam $\mathrm{P}^{(1)}$ is free at point A along an arc of a circle. Beam $\mathrm{P}^{(2)}$ is also clamped at its support (4) and free at its other extremity. A velocity $V$ along the $x$-axis is imposed on the support of beam $\mathrm{P}^{(2)}$ by assembly $(4)+(8)$.

In this experimental part, the friction-induced vibrations of two beams in contact whose dimensions are given in Table 1 are stud-

a

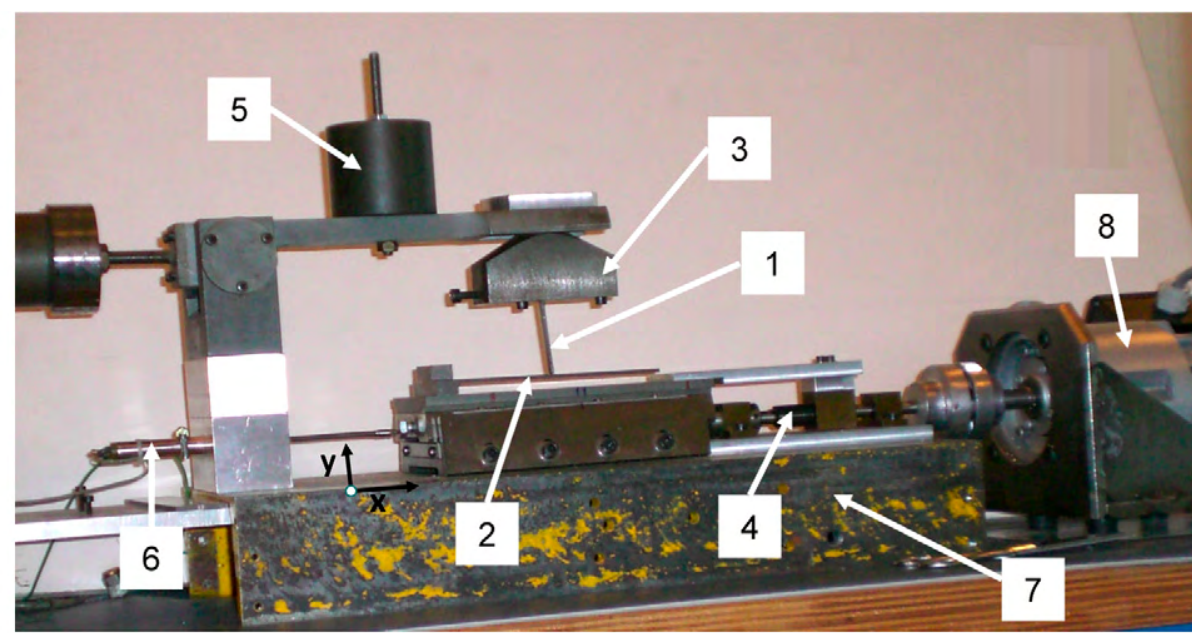

b

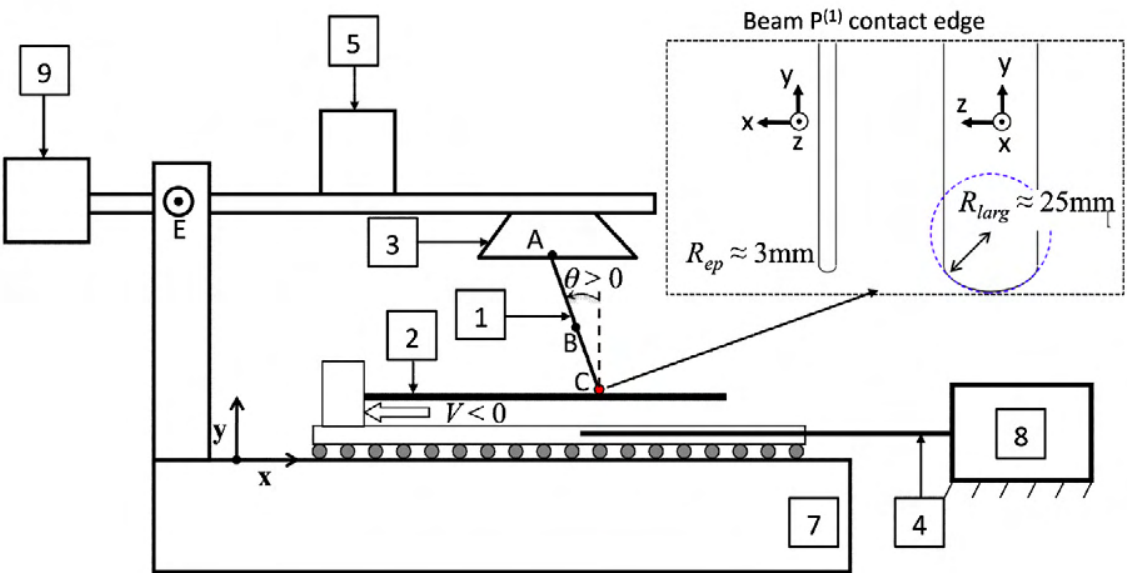

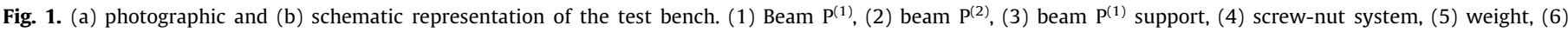
displacement transducer, (7) frame, (8) DC motor, and (9) counterweight. 
Table 1

Fixed data of the two beams $\mathrm{P}^{(1)}$ and $\mathrm{P}^{(2)}$.

\begin{tabular}{lll}
\hline & $\mathrm{P}^{(1)}(\mathrm{m})$ & $\mathrm{P}^{(2)}(\mathrm{m})$ \\
\hline Dimensions & 0.05 & 0.15 \\
Length & 0.01 & 0.015 \\
Width & 0.0015 & 0.003 \\
Thickness & & \\
Material & Steel & Steel \\
\hline
\end{tabular}

ied. This test rig has been designed to reduce the number of parameters playing a role in friction-induced vibrations. The contact zone on beam $\mathrm{P}^{(1)}$ has been reduced to avoid the edge effects influencing the dynamics of the system, by designing the contact edge of beam $\mathrm{P}^{(1)}$ as an arc of circle in the $z$ - and $y$-directions (Fig. 1b). The design of the mechanism (beam supports) is rather massive to avoid interactions with the dynamics of the two beams. The DC motor (8) can impose a uniform velocity $V$ from a value of $1 \mathrm{~mm} / \mathrm{s}$ to $5 \mathrm{~mm} / \mathrm{s}$.

\subsection{Study of vibrations of the system when beam $P^{(2)}$ is in translation}

During a specific test (in sliding steady state $(V>0)$ with no friction-induced vibrations), the Coulomb friction coefficient $\mu$ was measured at 0.2 . In order to control experimental contact conditions, an experimental process (including thorough cleaning of the surfaces) was defined to obtain reproducible contact conditions. For each test presented this experimental process was applied and repeated three times to ensure the reproducibility of the results. The beams were placed in contact and when the system was in static equilibrium, beam $\mathrm{P}^{(2)}$ was set in motion. The measurements of velocity of point B (Fig. 1) in the $x$-direction $\left(v_{B}(t)=\dot{u}_{1}^{(1)}\left(\mathbf{x}_{B}, t\right)\right)$ were performed during this latter phase. A laser Doppler vibrometer was used to measure velocity without contact so as not to influence the dynamics of the system.

The measured velocity $v_{B}(t)$ for translation velocities $V$ of $-1 \mathrm{~mm} / \mathrm{s},-2 \mathrm{~mm} / \mathrm{s}$, and $-5 \mathrm{~mm} / \mathrm{s}$ is shown in Fig. 2. The system exhibited periodic steady state vibrations $(V=-5 \mathrm{~mm} / \mathrm{s})$ and intermittent periodic steady state vibrations $(V=-1 \mathrm{~mm} / \mathrm{s} ; V=-2 \mathrm{~mm} /$ s). Fig. 3 shows the FFT of the measured vibrations of Fig. 2 . For the three imposed velocities the frequency spectrum is periodic characterized by one main frequency $2700 \mathrm{~Hz}$ and its harmonics $5400 \mathrm{~Hz}$ and $8100 \mathrm{~Hz}$. Other frequencies appeared $(2080 \mathrm{~Hz}$, $6500 \mathrm{~Hz}$ ) in the spectrum. Note that even if the vibrating phenomenon is quite different (periodic steady state and intermittent periodic steady state vibrations) the fundamental frequency

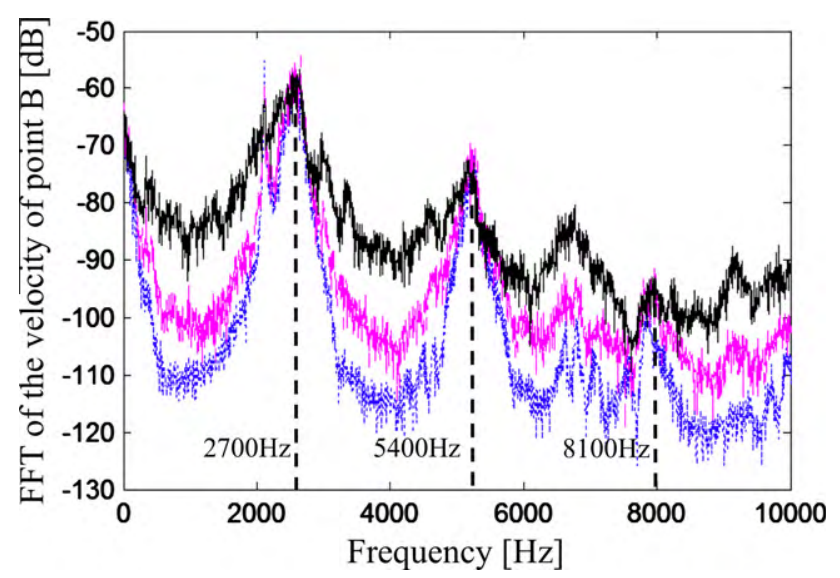

Fig. 3. FFT of measured velocities of $v_{B}(t)$ for translation velocities $V$ of $(\ldots . .)-$. $1 \mathrm{~mm} / \mathrm{s},(-)-2 \mathrm{~mm} / \mathrm{s}$ and $(-)-5 \mathrm{~mm} / \mathrm{s}\left(F=9 \mathrm{~N}\right.$ and $\left.\theta=5^{\circ}\right)$. (For interpretation of the references to colour in this figure legend, the reader is referred to the web version of this article.)

corresponds to the same eigenmode of the system. This intermittent periodic steady state vibrations corresponds to a succession of two different periods of time: one of high level vibrations (dur$\operatorname{ing} T_{v i b}$ ) (whose fundamental frequency is $2700 \mathrm{~Hz}$ ) and one during which the vibrations attenuated and tended to vanish (during $T_{S t}$ ). The average of $T_{S t}$ is inversely proportional to the velocity imposed $V$ (Fig. 2). Above a certain velocity, $T_{S t}$ tends to vanish and the system vibrated without intermittence.

To understand these vibrating phenomena and the dynamic contact behaviour, a numerical study was performed. These studies are based on two types of analysis: complex eigenvalue analysis (linear) and dynamic transient analysis (non-linear).

\section{Numerical study}

\subsection{Model of the experimental system}

Fig. 4 shows the model of the experimental system presented in Fig. 1. The two steel beams $\mathrm{P}^{(1)}$ and $\mathrm{P}^{(2)}$ are in frictional contact. The contact surfaces are considered as being perfectly flat. The thermal and physicochemical effects are neglected. The mechanism governing $\mathrm{P}^{(1)}$ and $\mathrm{P}^{(2)}$ is modeled as a boundary condition. $\mathrm{P}^{(2)}$ is free-clamped. Point A of $\mathrm{P}^{(1)}$ is blocked in translation in the $x$-direc-
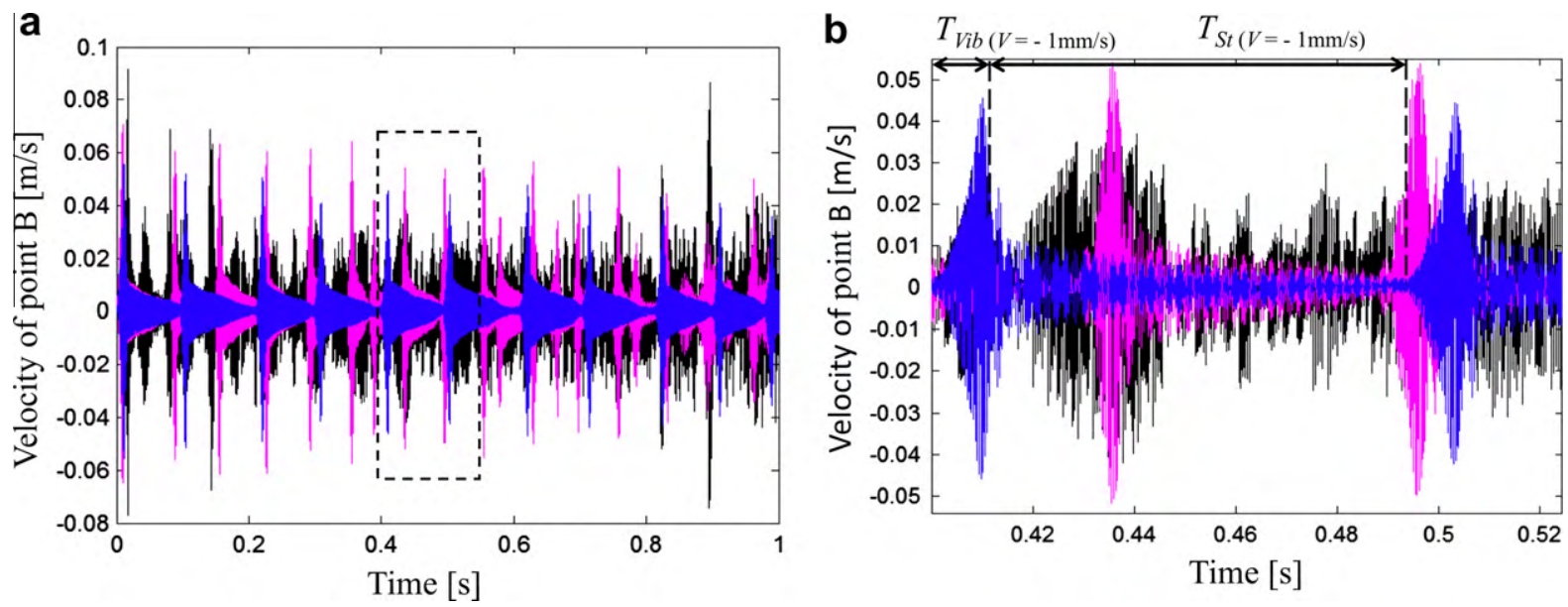

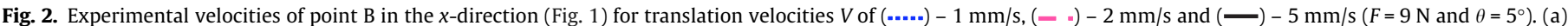

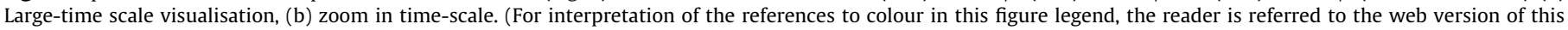
article.) 


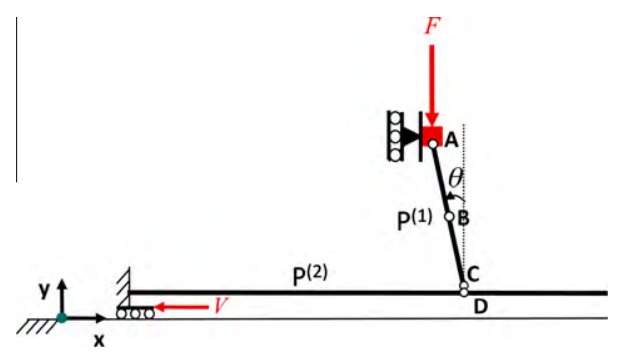

Fig. 4. Representation of the model system studied. Initially, point $C$ is $80 \mathrm{~mm}$ from the clamp of beam $\mathrm{P}^{(2)}$ for the numerical simulations and tests described. $\mathrm{D}$ is the contact node on $\mathrm{P}^{(2)}$, defined according to the closest projection of node $\mathrm{C}$ to $\mathrm{P}^{(2)}$. $\square$ ): Concentrated mass. For the reference case: $V=-2 \mathrm{~mm} / \mathrm{s}, F=9 \mathrm{~N}$, and $\theta=5^{\circ}$. (For interpretation of the references to colour in this figure legend, the reader is referred to the web version of this article.)

tion and in rotation in the $z$-direction. The assembly $(3)+(9)+(5)$ is modelled by an equivalent concentrated mass whose value was determined during the study of the dynamics of the system (with $V=0 \mathrm{~mm} / \mathrm{s}$ ) (see further). A force $F$ was applied at point $A$ in the $y$ direction, corresponding to the force applied by the weight (5). A velocity $V$ in the $x$-direction was imposed at $\mathrm{P}^{(2)}$ at the clamp.

\subsection{The governing equations}

The deformation of two elastic bodies occupying two domains $\left(\mathrm{P}^{(\mathrm{L})}\right)_{L=1,2}$ in the initial configuration is considered. It should be noted that the superscripts of the form $(\cdot)^{(\mathrm{L})}, L=1,2$ denote association with either of the two bodies. The system under consideration is described in terms of material frame. The current Cartesian component of a point are $\mathbf{x}=\left(x_{1}, x_{2}\right)$ (Fig. 4). The boundary $\Gamma^{(\mathrm{L})}$ of each solid is the union of three non-overlapping subregions $\Gamma^{(\mathrm{L})}=\Gamma_{\mathrm{u}}^{(\mathrm{L})} \cup \Gamma_{\mathrm{g}}^{(\mathrm{L})} \cup \Gamma_{\mathrm{c}}^{(\mathrm{L})}$. The displacement field $\overline{\mathbf{u}}^{(\mathrm{L})}=\left(\bar{u}_{1}^{(\mathrm{L})}, \bar{u}_{2}^{(\mathrm{L})}\right)$ is imposed on $\Gamma_{\mathrm{u}}^{(\mathrm{L})}$, surface traction $\bar{t}^{(\mathrm{L})}$ is applied on $\Gamma_{\mathrm{g}}^{(\mathrm{L})}$. The remainder of $\Gamma^{(\mathrm{L})}, \Gamma_{c}^{(\mathrm{L})}$ is selected so that all expected nodes of contact with the opposing body are included. The $\mathrm{P}^{(\mathrm{L})}$ body is subjected to body force $\boldsymbol{f}$.

To observe the impenetrability condition $\left(\Gamma_{c}^{(1)} \cap \Gamma_{c}^{(2)}=0\right)$, it is convenient to consider $\Gamma_{c}^{(1)}$ as the slave surface (restricted to node C) and $\Gamma_{c}^{(2)}$ as the target surface. At time $t$, contact node $\mathrm{D} \in \Gamma_{c}^{(2)}$ is identified, according to the closest projection of node $\mathrm{C}$ to $\Gamma_{c}^{(2)}$ :

$\mathbf{x}_{D}\left(\mathbf{x}_{C}, t\right)=\min _{\mathbf{x}^{(2)} \in \Gamma_{c}^{(2)}}\left\|\mathbf{x}_{C}-\mathbf{x}^{(2)}\right\|_{2}$

A local coordinate system $(\mathbf{t}, \mathbf{n})$ is defined at each node of the target contact surface $\Gamma_{c}^{(2)}$. By defining $\mathbf{n}=\left(n_{1}, n_{2}\right)$ as the inward unit vector normal at node $\mathrm{D}$, the initial gap function $g\left(\mathbf{x}_{C}\right)$ (or gap at zero displacement) between $\Gamma_{c}^{(1)}$ and $\Gamma_{c}^{(2)}$ is $g\left(\mathbf{x}_{C}\right)=g=\left(\mathbf{x}_{D}-\mathbf{x}_{C}\right)^{T}$. n. If $\sigma$ denotes the Cauchy stress at node $C$, then $\boldsymbol{\sigma}_{N}\left(\mathbf{x}_{C}\right)=\sigma_{i j}\left(\mathbf{x}_{C}\right) n_{i} n_{j}$, $1 \leqslant i, j \leqslant 2$ and $\boldsymbol{\sigma}_{T}\left(\mathbf{x}_{C}\right)=\sigma\left(\mathbf{x}_{C}\right) \cdot \mathbf{n}-\sigma_{N}\left(\mathbf{x}_{C}\right) \mathbf{n}$ denotes its normal and tangential contact stresses.

For linear elasticity, the general Signorini problem with Coulomb friction consists in finding the displacement fields $\mathbf{u}^{(\mathrm{L})}=\left(u_{1}, u_{2}\right)$ satisfying the system of equalities and inequalities:

$\sigma_{i j}=E_{i j k l}^{(\mathrm{L})} \frac{\partial u_{k}}{\partial x_{l}}=E_{i j k l}^{(\mathrm{L})} u_{k, l}^{(\mathrm{L})} \quad$ in $\mathrm{P}^{(\mathrm{L})}$

$\sigma_{i j}^{(\mathrm{L})}\left(\mathbf{u}^{(\mathrm{L})}\right)_{j}+f_{i}=\rho \ddot{u}_{i}^{(\mathrm{L})} \quad$ in $\mathrm{P}^{(\mathrm{L})}$

$\sigma_{i j}\left(\mathbf{u}^{(\mathrm{L})}\right) n_{j}^{(\mathrm{L})}=\bar{t}_{i}^{(\mathrm{L})} \quad$ on $\Gamma_{g}^{(L)}$

$u_{i}^{(L)}=\bar{u}_{i}^{(L)} \quad$ on $\Gamma_{u}^{(L)}$

$\sigma_{N}\left(\mathbf{x}_{C}\right)=0$ and $\sigma_{T}\left(\mathbf{x}_{C}\right)=\mathbf{0}$ if $u_{N}<g$ on $\Gamma_{c}^{(1)}$

If $u_{N}=g$ then $\sigma_{N}\left(\mathbf{x}_{C}\right)<0$ and if, in addition

$\left\|\sigma_{T}\left(\mathbf{x}_{C}\right)\right\|<\mu\left|\sigma_{N}\left(\mathbf{x}_{C}\right)\right|$ then $\dot{\mathbf{u}}_{T}=\mathbf{0}$ (Stick) while

if $\left\|\boldsymbol{\sigma}_{T}\left(\mathbf{x}_{C}\right)\right\|=\mu\left|\sigma_{N}\left(\mathbf{x}_{C}\right)\right|$ then $\exists \zeta>0$ such that $\dot{\mathbf{u}}_{T}=-\zeta \sigma_{T}\left(\mathbf{x}_{C}\right)$ (Slip) where $E_{i j k l}^{(\mathrm{L})}$ are the components of Hooke's tensor, $\rho$ represents the density, the double dot stands for the second partial derivative relative to time, $u_{N}\left(\mathbf{x}_{C}\right)=u_{N}=\left(\mathbf{u}^{(1)}\left(\mathbf{x}_{C}\right)-\mathbf{u}^{(2)}\left(\mathbf{x}_{D}\right)\right)^{\mathrm{T}} \cdot \mathbf{n}$ is the rate of interpenetration of the two bodies [22], $\dot{\mathbf{u}}_{T}=\frac{\partial\left(\mathbf{u}^{(1)}\left(\mathbf{x}_{C}\right)-\mathbf{u}^{(2)}\left(\mathbf{x}_{D}\right)-u_{N}\left(\mathbf{x}_{C}\right) \mathbf{n}\right)}{\partial t}$ represents the relative tangential velocity through $\boldsymbol{\Gamma}_{c}$ and $\mu$ is the Coulomb friction coefficient. Eqs. (1)-(5) respectively designate the constitutive relation, the linear momentum balance, the Neumann and Dirichlet conditions and non-contact. Eq. (6) asserts that if contact is made, then no sliding of node $C$ occurs if the magnitude of $\left\|\sigma_{T}\left(\mathbf{x}_{C}\right)\right\|$ is below a critical value, and when $\left\|\sigma_{T}\left(\mathbf{x}_{C}\right)\right\|$ reaches this critical level, a tangential slip occurs in a direction opposite to $\boldsymbol{\sigma}_{T}\left(\mathbf{x}_{C}\right)$.

For this model the discrete formulation with Lagrange multipliers is used. Spatial discretization is performed with the finite elements methods. The elements considered are Euler-Bernoulli beam elements (shearing neglected) in bending and traction-compression $\left(3^{\circ}\right.$ of freedom per node). Linear hypotheses of small rotations and small deformations are used. The contact between the two beams is a node-on-segment contact condition [23].

The finite element solution of the governing continuum mechanics equations is obtained by using the discretization procedures for the principle of virtual work and by discretizing the contact conditions at node C [24], yielding:

$$
\left\{\begin{array}{l}
\mathbf{M U}+\mathbf{C} \dot{\mathbf{U}}+\mathbf{K U}+\mathbf{G}^{\mathrm{T}} \lambda=\mathbf{F} \\
\mathbf{G U} \leqslant 0
\end{array}\right.
$$

where $\mathbf{M}, \mathbf{C}$, and $\mathbf{K}$ are respectively symmetric and positive definite matrices of mass, Rayleigh's proportional damping $\left(C=d_{1} \mathbf{M}+d_{2} \mathbf{K}\right)$ and stiffness of the system. $\mathbf{U}, \dot{\mathbf{U}}, \ddot{\mathbf{U}}$ are respectively the vectors of nodal displacements, nodal velocities and nodal accelerations. $\mathbf{F}$ is the vector of nodal external forces.

$\lambda=\left[\lambda_{N} \lambda_{T}\right]^{\mathrm{T}}$ contains respectively normal and tangential forces at contact point C. $\mathbf{G}^{\mathrm{T}}=\left[\mathbf{G}_{N}^{\mathrm{T}} \mathbf{G}_{T}^{\mathrm{T}}\right]$ is the global matrix of the displacement conditions ensuring non-penetration and the contact law of the bodies in contact (see Appendix A). $\mathbf{G}_{N}$ and $\mathbf{G}_{T}$ have order $1 \times N d o f$, where Ndof is the number of degrees of freedom.

The characteristics of the modelled system and operating conditions are given in Tables 2 and 3. For the results presented, the contact between the two beams occurs at a point of beam $\mathrm{P}^{(2)}$ situated at $80 \mathrm{~mm}$ from the clamp (Fig. 4).

\subsection{Dynamics of the system $(V=0 \mathrm{~mm} / \mathrm{s})$}

Before beginning the numerical study of friction-induced vibrations, the authors first verified that the dynamics of the system at

Table 2

Operating conditions for the performed tests.

\begin{tabular}{ll} 
Applied force $(F)$ & $9 \mathrm{~N}$ \\
Applied velocity $(V)$ & $-1 ;-2 ;-5 \mathrm{~mm} / \mathrm{s}$ \\
Coefficient of friction $(\mu)$ & 0.2 (measured) \\
Angle $\theta$ & $5^{\circ}$ \\
\hline
\end{tabular}

Table 3

Fixed data of the numerical model of the system studied.

\begin{tabular}{lll}
\hline & $\mathrm{P}^{(1)}$ & $\mathrm{P}^{(2)}$ \\
\hline Length & $0.05 \mathrm{~m}$ & $0.15 \mathrm{~m}$ \\
Width & $0.01 \mathrm{~m}$ & $0.015 \mathrm{~m}$ \\
Thickness & $0.0015 \mathrm{~m}$ & $0.003 \mathrm{~m}$ \\
Density $(\rho)$ & $7900 \mathrm{~kg} / \mathrm{m}^{3}$ & $7900 \mathrm{~kg} / \mathrm{m}^{3}$ \\
Young's modulus $(E)$ & $185 \mathrm{GPa}$ & $185 \mathrm{GPa}$ \\
Parameter $d_{1}$ & $50 \mathrm{~s}^{-1}$ & $50 \mathrm{~s}^{-1}$ \\
Parameter $d_{2}$ & $1 \times 10^{-8} \mathrm{~s}$ & $1 \times 10^{-8} \mathrm{~s}$ \\
Number of elements & 30 & 90 \\
\hline
\end{tabular}


rest $(V=0 \mathrm{~mm} / \mathrm{s})$ was modelled correctly. Experimental modes and natural frequencies of the system (with the two beams stuck by glue at contact) were determined in order to compare them to those calculated numerically. The experimental natural frequencies were determined by applying white noise to the system and then the experimental modes were obtained by measuring the vibratory amplitudes and phases of different points of the system subjected to sinusoidal excitation at experimental natural frequencies. These modes were then compared to numerical modes obtained by a classical search for the eigenmodes of two beams stuck at their contact. All the modes in the frequency range investigated $(f<10 \mathrm{kHz})$ obtained experimentally were identified. The concentrated mass in the numerical model was adjusted to ensure the correspondence between the numerical and experimental first natural frequencies equal to $16 \mathrm{~Hz}$. The experimental and numerical frequencies are given in Table 4 . The error on the frequencies does not exceed $4 \%$, thus validating the dynamic modelling of the experimental system presented in Fig. 1.

\subsection{Complex eigenvalue analysis}

In this analysis we focussed on the study of flutter instability of the steady sliding state. The gyroscopic contributions due to transport motion [25] are supposed to have no significant influence. This assumption is post hoc justified by the good correspondence between simulation and experiment. For recent work on the topic, please refer to [26].

\subsubsection{Flutter instability of the steady sliding state}

The following discussion focuses on the linear stability of the steady sliding equilibrium.

The first step is the determination of the steady sliding equilibrium. For this case of frictional contact problem, at steady sliding equilibrium $(\ddot{\mathbf{U}}=\dot{\mathbf{U}}=\mathbf{0})$ the slave node $\mathrm{C}$ of $\mathrm{P}^{(1)}$ is in sliding state $\left(g\left(\mathbf{x}_{C}\right)=0\right)$. The contact tangential force is known: $\lambda_{T} \mathbf{t}=\mu \lambda_{N} \mathbf{t}\left(\lambda_{N} \leqslant 0\right)$. Thus the system (7) becomes:

$$
\left\{\begin{array}{l}
\mathbf{K} \mathbf{U}^{e}+\mathbf{G}_{N}^{\mathrm{T}} \lambda_{N}=\mathbf{f}-\mu \lambda_{N} \mathbf{t} \mathbf{G}_{T}^{\mathrm{T}} \\
\mathbf{G}_{N} \mathbf{U}^{\mathrm{e}}=0
\end{array}\right.
$$

where $\mathbf{U}^{\mathrm{e}}$ is the vector of nodal displacement of the system at steady sliding equilibrium.

Once the steady sliding equilibrium is determined, attention is given to the possible existence of a dynamic solution in the neighbourhood of this equilibrium state. A perturbed dynamic solution is considered in the neighbourhood of this steady sliding equilibrium, such that the contact point remains in sliding state. From the expressions $\mathbf{U}=\mathbf{U}^{\mathrm{e}}+\widehat{\mathbf{U}}$ and $\lambda_{N}=\lambda_{N}^{\mathrm{e}}+\hat{\lambda}$, the dynamic problem may be written:

$$
\left\{\begin{array}{l}
\mathbf{M} \ddot{\hat{\mathbf{U}}}+\mathbf{C} \dot{\hat{\mathbf{U}}}+\mathbf{K} \ddot{\hat{\mathbf{U}}}+\mathbf{G}_{N}^{\mathrm{T}} \hat{\lambda}_{N}=-\mu \frac{\dot{\mathbf{u}}_{t}}{\left\|\mathbf{u}_{t}\right\|} \mathbf{G}_{T}^{\mathrm{T}} \hat{\lambda}_{N} \\
\mathbf{G}_{N} \widehat{\mathbf{U}}=0
\end{array}\right.
$$

Table 4

Numerical and experimental natural frequencies and relative error.

\begin{tabular}{lll}
\hline $\begin{array}{l}\text { Numerical } \\
\text { frequencies }(\mathrm{Hz})\end{array}$ & $\begin{array}{l}\text { Experimental } \\
\text { frequencies }(\mathrm{Hz})\end{array}$ & $\begin{array}{l}\text { Relative error on the } \\
\text { frequency value }(\%)\end{array}$ \\
\hline 16 & 16 & 0 \\
362 & 374 & 3.2 \\
1665 & 1620 & 2.8 \\
2060 & 2080 & 0.9 \\
2924 & 2930 & 0.1 \\
5035 & 4875 & 3.3 \\
6624 & 6600 & 0.4 \\
8030 & 7690 & 4 \\
\hline
\end{tabular}

where $\wedge$ denotes the perturbed state from the steady sliding equilibrium. We assumed that the motion of node $C$ remains planar (2D). In this case, contact with friction does not induce supplementary terms in damping matrix [26,27] and the contact conditions $\lambda_{T}=\mu \frac{\mathbf{u}_{t}}{\left\|\mathbf{u}_{t}\right\|} \mathbf{G}_{T}^{\mathrm{T}} \lambda_{N}$ give, after linearization $\hat{\lambda}_{T}=\mu \mathbf{t G}_{T}^{\mathrm{T}} \hat{\lambda}_{N}$. It is then possible to express $\hat{\lambda}_{T}$ as a function of $\hat{\mathbf{U}}$ and $\widehat{\mathbf{U}}$ :

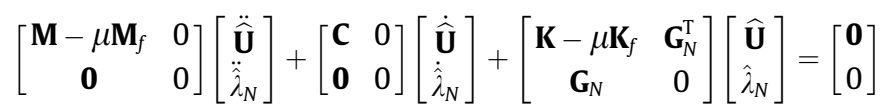

with $\mathbf{M}_{f}$ and $\mathbf{K}_{f}$ being matrices containing the supplementary terms due to tangential contact force. The expressions of these supplementary terms are detailed in [19,27].

The equations that govern the smooth dynamic evolution of the system are:

$\tilde{\mathbf{M}} \ddot{\mathbf{U}}_{T O T}+\tilde{\mathbf{C}} \dot{\mathbf{U}}_{T O T}+\tilde{\mathbf{K}} \mathbf{U}_{T O T}=\mathbf{0}$

where $\tilde{\mathbf{M}}$ and $\tilde{\mathbf{K}}$ are non-symmetric matrices and $\mathbf{U}_{\text {ТОт }}=\left[\begin{array}{c}\widehat{\mathbf{U}} \\ \hat{\lambda}_{N}\end{array}\right]$. The definiteness of $\tilde{\mathbf{M}}$ and $\tilde{\mathbf{K}}$ depends on the value of $\mu$ and $\theta$. Consequences on stability have been discussed in [28].

The linear analysis of the dynamic stability of the system (11) leads classically to the generalized eigenproblem:

$\left\{\varphi^{2} \tilde{\mathbf{M}}+\varphi \tilde{\mathbf{C}}+\tilde{\mathbf{K}}\right\} \psi=0$

The lack of symmetry leads to complex modes and complex eigenvalues. The complex and conjugate pairs of eigenvalues $\varphi_{i}$ can be written as $\varphi_{i}=-\eta_{i} \omega_{i}+j \omega_{i}$, where $\omega_{i}$ is the $i$ th angular frequency of the system. The existence of a non-trivial eigenvector for several $\varphi_{i}$ with a strictly positive real part $\left(\eta_{i}<0\right)$ implies the instability of the steady sliding equilibrium: a divergence instability if $\omega_{i}=0$ (non-oscillatory) and a flutter instability if $\omega_{i} \neq 0$ (oscillatory) [19]. The generalized eigenvalue problem (12) is solved by using the double QZ algorithm [29].

Attention is given here to flutter instability. It can be noted that a negative $\eta_{i}$ corresponds to negative equivalent damping coefficient.

\subsubsection{Complex eigenvalue analysis (CEA) of the system}

Fig. 5 shows the CEA of the system as a function of the friction coefficient. In the frequency domain considered $(<10 \mathrm{kHz})$ and for

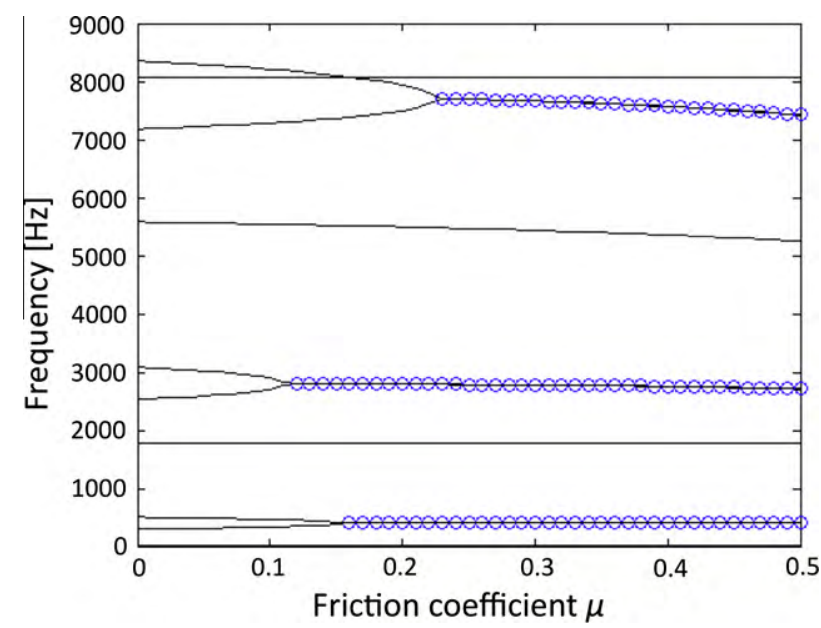

Fig. 5. Complex eigenvalue analysis of the system studied: evolution of the frequency of the 10 first modes of the system coupled by friction as a function of Coulomb coefficient of friction $\mu\left(F=9 \mathrm{~N}, \theta=5^{\circ}\right) . \bigcirc$ (blue) unstable frequencies. (For interpretation of the references to colour in this figure legend, the reader is referred to the web version of this article.) 
Coulomb friction coefficient $\mu$ between 0 and 0.5 , three mode coalescences $(400 \mathrm{~Hz}, 2800 \mathrm{~Hz}$, and $7700 \mathrm{~Hz})$ are observed at different critical friction coefficients: $\mu_{c}^{400 \mathrm{~Hz}}=0.16, \mu_{c}^{2800 \mathrm{~Hz}}=0.12$ and $\mu_{c}^{7700 \mathrm{~Hz}}=0.23$.

The CEA predicts two eigenvalues with $\eta_{i}<0$ at $\mu=0.2$. The corresponding frequencies are $400 \mathrm{~Hz}$ and $2800 \mathrm{~Hz}$. This second frequency $(2800 \mathrm{~Hz})$ corresponds to the experimental fundamental frequency of vibration $(2700 \mathrm{~Hz}$ Fig. 3$)$. This confirms that the observed vibrations of the system come from a flutter instability phenomenon predicted by this linear analysis.

In the case presented here the damping taken into account has a weak influence and the results with damping are very close to the results obtained without damping. Studies on effect of damping on flutter instability are presented in [30,31]. Fig. 6a shows the 5th and the 6th eigenvalues in the complex plane. For a friction coefficient $\mu$ equal to zero both are far from the other $\left(\omega_{5}=2500 \mathrm{~Hz}\right.$ and $\omega_{6}=3100 \mathrm{~Hz}$ ). By increasing friction coefficient $\mu$ eigenvalues 5 and 6 move parallel to the imaginary axis until the lock-in ( $\mu=0.12$ ) [32], the limit point of stability, where $\omega_{5} \approx \omega_{6} \approx$ $2800 \mathrm{~Hz}$. As the friction coefficient $\mu$ still increases, one eigenvalue crosses the imaginary axis and becomes unstable $\left(\eta_{6}<0\right)$ and the other eigenvalue moves to the increasing but positive apparent damping coefficient $\eta_{5}$ (stable eigenvalue). Fig. $6 \mathrm{~b}$ represents the unstable mode at the lock-in point. The deformed shape of this mode exhibits considerable coupling between tangential and normal vibrations at the contact, which is known to be an important parameter of friction-induced instability [20].

This linear analysis provides an approach, which can explain the experimentally observed vibration phenomena as flutter instability of the system due to the contact. It corresponds to a phenomenon of lock-in [20] of two structure assembly modes.

Indeed the experimental results showed complex vibrating behaviour as a function of imposed velocity $V$. To study the vibrations and the contact dynamics of the system during unstable behaviours and to complete linear analysis, a transient dynamic analysis that takes into account the non-linear aspects (stick, slip, separation) of contact with friction was performed.

3.5. Dynamic transient analysis: self-excited vibrations and limit-cycle of the system

3.5.1. Time domain scheme and equations of motion including contact with friction

The values of displacements, velocities and accelerations at the different nodes, as well as the values of the forces and contact node

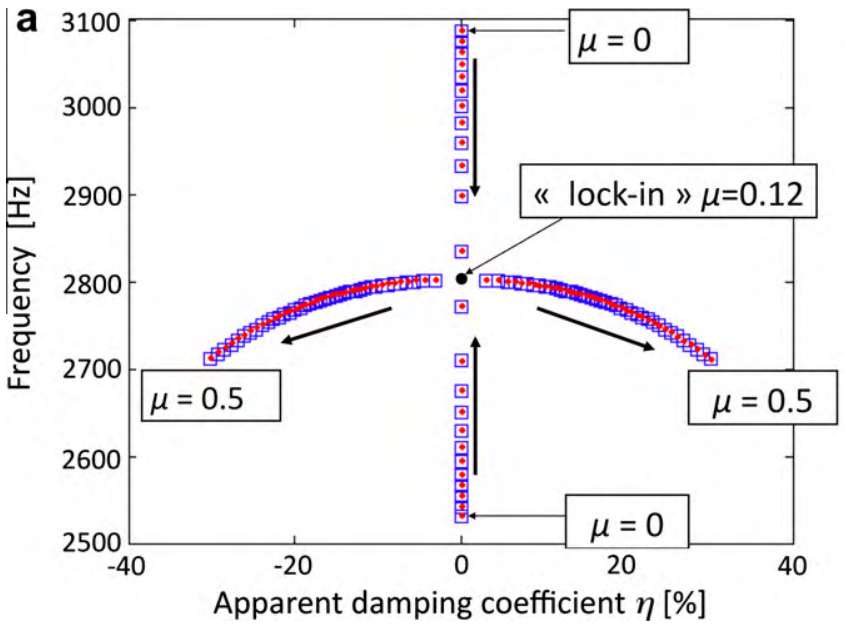

status (stick, slip, separation) are calculated through time. The frictional contact node between the two deformable beams is controlled by algorithms that use the Lagrange multiplier method [24] based on the interaction between the target segments and slave nodes. For the system under study, a slave node (node located at point $C$ belonging to $\mathrm{P}^{(1)}$ ) and target segments (on $\mathrm{P}^{(2)}$ ) that can potentially be in contact with the slave node during the calculation are defined. The elementary target segments are described by two nodes and approximated by bicubic splines.

The forward Lagrange multiplier method is formulated by equations of motion (7) at time $\left(t^{i}=i \Delta t\right)$ with the displacement conditions imposed on the slave node at time $t^{i+1}$ :

$$
\left\{\begin{array}{l}
\mathbf{M} \ddot{U}^{i}+\mathbf{C U}^{i}+\mathbf{K} \mathbf{U}^{i}+\mathbf{G}^{i+1}{ }^{\mathrm{T}} \lambda^{i}=\mathbf{F}^{i} \\
\mathbf{G}^{i+1} \mathbf{U}^{i+1} \leqslant 0
\end{array}\right.
$$

The equations of motion (13) are discretized in time, by using an explicit Newmark scheme. The vectors $\ddot{\mathbf{U}}^{i}$ and $\dot{\mathbf{U}}^{i}$ are expressed at each time step using a time scheme of type $\beta_{2}\left(\beta_{2} \in[0.5 ; 1]\right)$ :

$$
\left\{\begin{array}{l}
\ddot{\mathbf{U}}^{i}=\frac{2}{\Delta t^{2}}\left(\mathbf{U}^{i+1}-\mathbf{U}^{i}-\Delta t \dot{\mathbf{U}}^{i}\right) \\
\dot{\mathbf{U}}^{i}=\frac{1}{1+2 \beta_{2}}\left\{\dot{\mathbf{U}}^{i+1}+\Delta t\left(1-\beta_{2}\right) \ddot{\mathbf{U}}^{i-1}+\frac{2 \beta_{2}}{\Delta t}\left(\mathbf{U}^{i+1}-\mathbf{U}^{i}\right)\right\}
\end{array}\right.
$$

The displacements ${ }^{*} \mathbf{U}^{i+1}$ of the nodes situated on the contact surface $\left(\mathrm{P}^{(1)}\right.$ and $\left.\mathrm{P}^{(2)}\right)$ are first computed with $\lambda^{i}$ equal to $\mathbf{0}$. For simplicity $\beta_{2}$ is fixed to 0.5 and the nodal displacements at time ${ }^{*} t^{i+1}$ are obtained so that:

${ }^{*} \mathbf{U}^{i+1}=\Delta t^{2} \mathbf{M}^{-1}\left(\mathbf{F}^{i}-\mathbf{K} \mathbf{U}^{i}\right)+2 \mathbf{U}^{i}-\mathbf{U}^{i-1}$

A constraint matrix $\mathbf{G}^{i+1}$ is formulated for the slave node if it has penetrated through a target segment. Calculations of contact forces $\lambda^{i}$ and nodal displacement $\mathbf{U}^{i+1}$ at time $t^{i+1}$ are then performed:

$\left\{\begin{array}{l}\lambda^{i}=\left\{\Delta t^{2} \mathbf{G}^{i+1} \mathbf{M}^{-1} \mathbf{G}^{i+1}{ }^{\mathrm{T}}\right\}^{-1} \mathbf{G}^{i+1}\left({ }^{*} \mathbf{U}^{i+1}\right) \\ \mathbf{U}^{i+1}={ }^{*} \mathbf{U}^{i+1}-\left(\Delta t^{2} \mathbf{M}^{-1} \mathbf{G}^{i+1}{ }^{\mathrm{T}} \lambda^{i}\right)\end{array}\right.$

Eq. (16) are solved using the Gauss-Seidel method. In the contact zone, the friction law used is a Coulomb type law with regularisation of the tangential force with the velocity [33,34]. The parameters of this law were chosen numerically to ensure convergence through grid size and time step reductions and without any influence of the physical phenomena. For clarity, the equations are presented with a classical Coulomb type law expressed in Eq. (6) and the Prakash-Clifton type law is used in computation (see Appendix B, [33,34]).

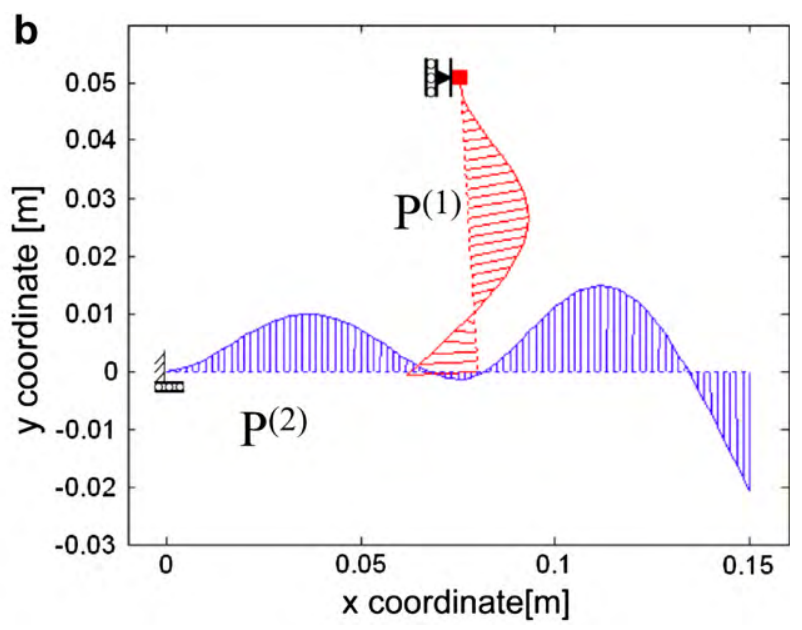

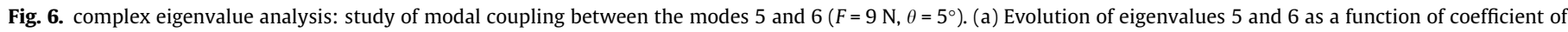
friction $\mu$. (b) Representation of unstable mode at lock-in. 


\subsubsection{Time domain simulations}

The parameters of the simulations presented are given in Tables 2, 3 and 5. The mesh and time step parameters were defined in order to obtain convergence through grid size and time step reductions. At initial time $(t=0 \mathrm{~s})$, the beams in contact are in static equilibrium. For $t>0 \mathrm{~s}$ a negative velocity is applied in the $x$-direction at $\mathrm{P}^{(2)}$. As the one experimentally measured, the desired constant value $V$ is reached after a linear ramp whose slope has no influence on the periodic steady state vibrations.

Table 5

Numerical parameters of the time simulations.

$\begin{array}{ll}\text { Time step }(\Delta t) & 2 \times 10^{-8} \mathrm{~s} \\ \text { Numerical damping }\left(\beta_{2}\right) & 0.8\end{array}$

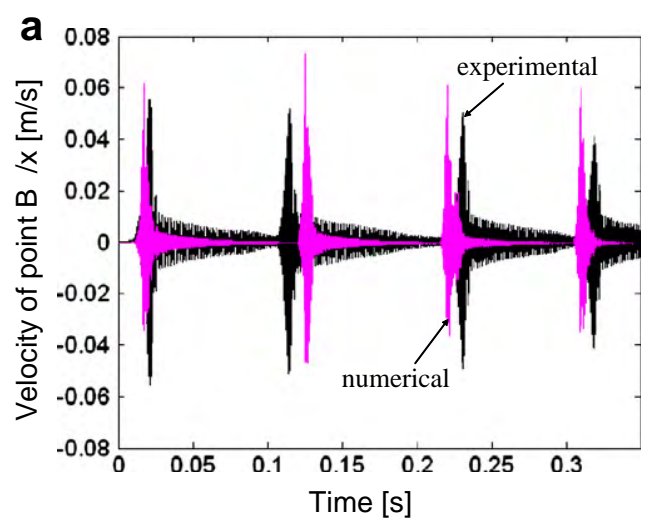

C

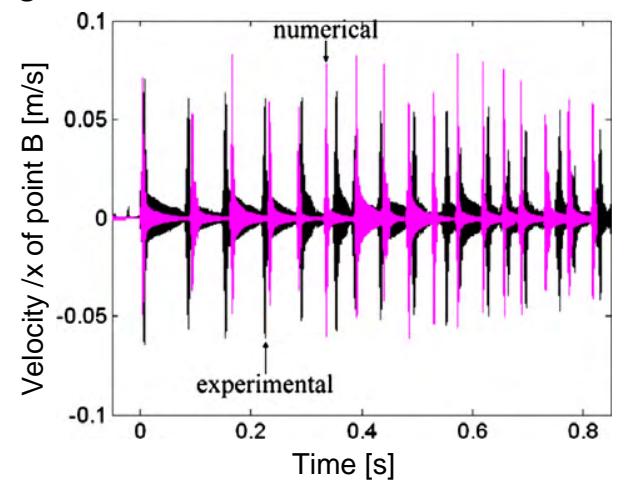

e

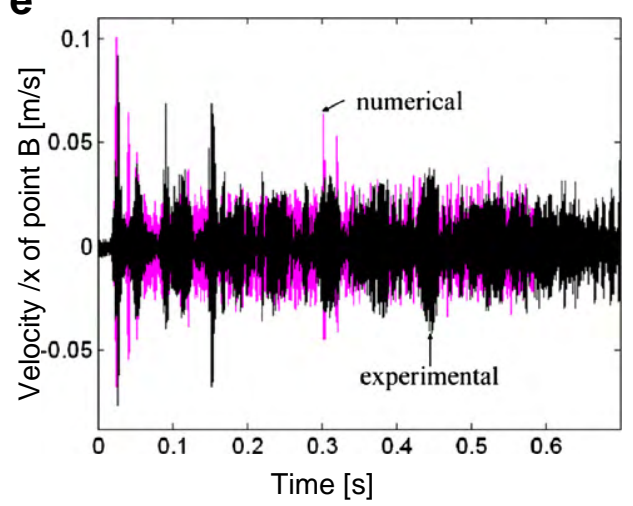

For $\mu \leqslant 0.12$, the system is in steady sliding state. To draw a parallel with linear analysis, this state is considered as a stable configuration. The relative tangential velocity and force values at the contact tend toward the imposed values. On the contrary $(\mu>0.12)$ the system exhibits important vibrations induced by flutter instability $[15,17,21,35]$. In this case the system is in an unstable configuration and tends to a limit cycle characterized by normal and rotational oscillations [35].

\subsubsection{Simulations of the dynamics of the system in the experimental} configurations

Fig. 7 shows experimental and numerical velocities of point B in time and in frequency for three values of imposed velocity $V$ $(-1 \mathrm{~mm} / \mathrm{s},-2 \mathrm{~mm} / \mathrm{s}$, and $-5 \mathrm{~mm} / \mathrm{s})$. As in experimental results the system exhibits numerically periodic steady state vibrations
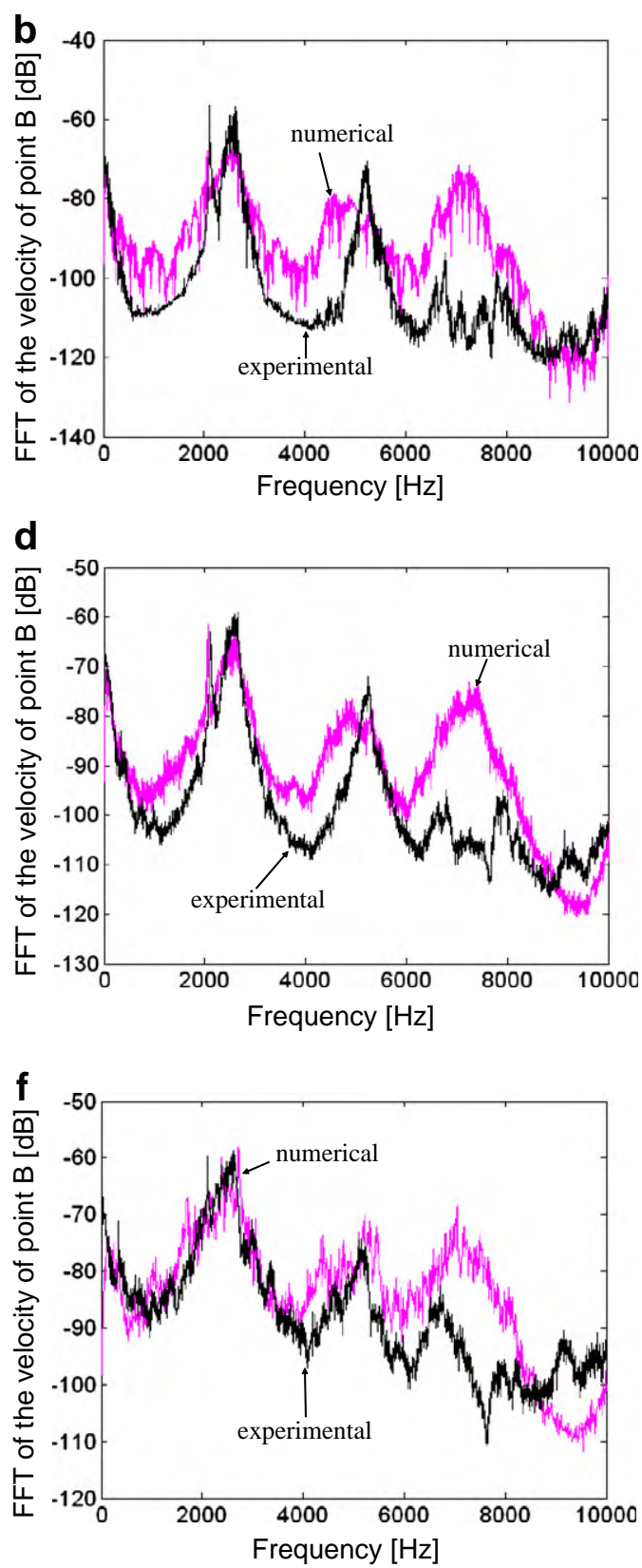

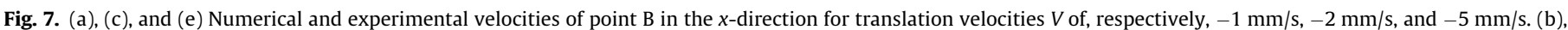

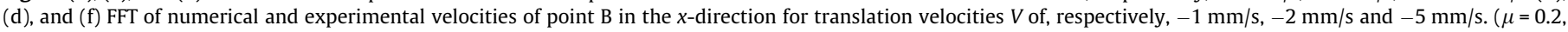
$F=9 \mathrm{~N}$ and $\theta=5^{\circ}$.) 
$(V=-5 \mathrm{~mm} / \mathrm{s})$ and intermittent periodic steady state vibrations $(V=-1 \mathrm{~mm} / \mathrm{s} ; V=-2 \mathrm{~mm} / \mathrm{s})$. This numerical analysis predicts the experimental trends observed previously. In spite of certain minimal differences that can be explained essentially by the fact that certain parameters are not taken into account (the proper dynamics of the experimental mechanism, the variability of the friction coefficient, surface roughness, etc.), the numerical and experimental signals in time are close to each other.

Fig. 7 also shows the numerical and experimental FFT of the velocity of point $B$ in the $x$-direction (for the imposed velocities of $-5 \mathrm{~mm} / \mathrm{s},-2 \mathrm{~mm} / \mathrm{s}$ and $-1 \mathrm{~mm} / \mathrm{s}$ ). According to these figures, both experimentally and numerically the vibrations of the system are periodic in terms of frequency, with a fundamental frequency of $2700 \mathrm{~Hz}$ (Fig. 7). The difference between the fundamental frequency of experimental and non-linear vibrations $(2700 \mathrm{~Hz})$ and the frequency obtained by stability analysis $(2800 \mathrm{~Hz})$ is due to the contact conditions. In linear analysis the sliding contact is imposed (no separation and no stick), whereas experimentally or in transient analysis the contact point of $\mathrm{P}^{(1)}$ can be in stick or in separated states. From both analyses modal shapes of the system are the same at this frequency.

The dynamic transient analysis therefore accurately predicts the frequency of the instability and its amplitude of vibrations. This latter analysis gives the amplitude of vibrations and the whole frequency content of vibratory response. The frequencies $2070 \mathrm{~Hz}$ and $6050 \mathrm{~Hz}$ could correspond to the 4th $(1800 \mathrm{~Hz})$ and 7 th modes $(5500 \mathrm{~Hz}$ ) of the system (Fig. 5). These modes are predicted stable by linear analysis and would be excited during instability due to contact dynamics. These supplementary frequencies could also be explained by complex combination of unstable frequencies due to strong contact non-linearity. To have more information on the origin of these frequencies it would be interesting to project the nodal displacements on the complex modal basis [36]. This point is not discussed in this paper.

Transient analysis is not only an accurate predictive model but also a means of investigating contact dynamics during instability.

\subsubsection{Analysis of numerical results}

3.5.4.1. Study of periodic steady state vibrations $(V=-5 \mathrm{~mm} / \mathrm{s})$. For an imposed friction coefficient $\mu=0.2$, Fig. 8 shows the time evolution of the normal contact force $\lambda_{N}$ and the apparent friction coefficient during two periods of vibration. The apparent friction coefficient [37] expressed by:

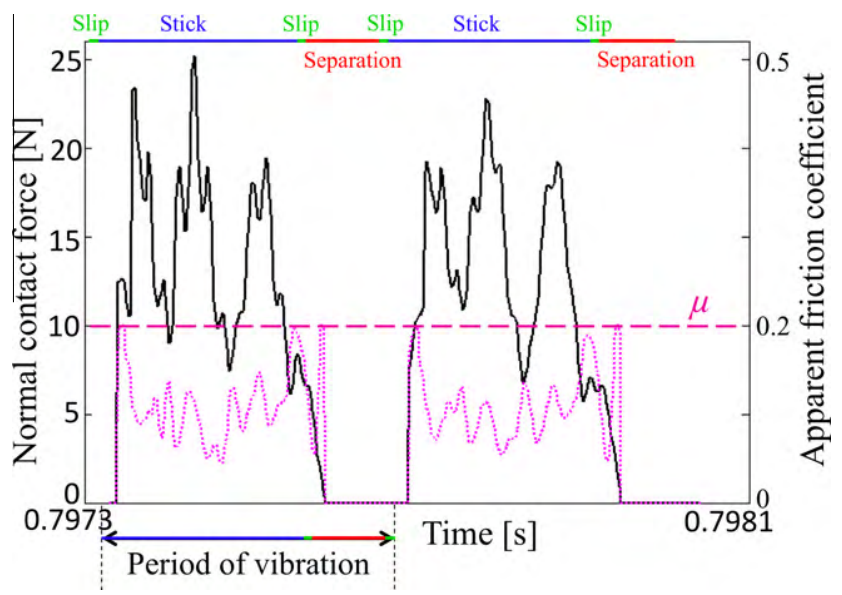

Fig. 8. Normal contact force and apparent coefficient of friction versus time for two periods of vibration. The contact slave node is successively in contact states of contact stick, contact slip, contact separation and contact slip during two periods of vibration. (Numerical time domain simulation with $\mu=0.2, V=-5 \mathrm{~mm} / \mathrm{s}, F=9 \mathrm{~N}$, and $\left.\theta=5^{\circ}\right)$. $\mu_{\text {apparent }}=\left\{\begin{array}{l}\left|\frac{\lambda_{T}}{\lambda_{N}}\right| \quad \text { if } \lambda_{N} \neq 0 \\ 0 \quad \text { if } \lambda_{N}=0\end{array}\right.$

gives the contact node status (stick, slip, separation) during a limit cycle. Indeed, when the system is sliding $\left(\left|\lambda_{T}\right|=\mu\left|\lambda_{N}\right|\right)$, both apparent and imposed coefficients of friction are equal [37]. As the system is in sticking state $\left(\left|\lambda_{T}\right|<\mu\left|\lambda_{N}\right|\right)$ the apparent coefficient of friction is less than the imposed coefficient of friction.

Fig. 9 shows relative velocity in the $x$-direction versus the displacement in the $x$-direction of node $C$ during a period of vibration (period of Fig. 8). Both Figs. 8 and 9 show four successive states of the slave node: a contact slip phase, a contact stick phase, a contact slip phase and a separation phase. During the separation phase, the normal contact force is null, since there is no contact between the two beams. During the slip phase, the apparent friction coefficient is equal to the imposed friction coefficient $(\mu=0.2)$ and the relative velocity at the contact can reach $30 \mathrm{~mm} / \mathrm{s}$, whereas the imposed velocity is $-5 \mathrm{~mm} / \mathrm{s}$. During the stick phase, the apparent friction coefficient is smaller than the imposed one. When the relative velocity at the contact is null, it can be seen that the normal contact force can reach $25 \mathrm{~N}$ instead of the $9 \mathrm{~N}$ imposed at point $\mathrm{A}$. Therefore, friction-induced vibrations involve more severe contact conditions.

3.5.4.2. Study of intermittent periodic steady state vibrations $(V=$ $-2 \mathrm{~mm} / \mathrm{s}$ ). Fig. 10 shows the contact force in the $y$-axis and the tangential slip velocity along time.

This intermittent phenomenon involves two phases: the system undergoes a phase of high level vibrations $\left(T_{v i b}\right)$ and a phase during which the vibrations attenuated and vanished $\left(T_{S t}\right)$ (Fig. 10b). During $T_{v i b}$, the system exhibits high-frequency vibrations at a frequency of $2700 \mathrm{~Hz}$ and the contact node successively sticks, slides and separates in comparison to the contact surface of $\mathrm{P}^{(2)}$. These vibrations are due to flutter instability of steady sliding equilibrium, as in Section 3.5.4.1. The normal contact force and the relative velocity between the two beams at the contact reach respectively $50 \mathrm{~N}(F=9 \mathrm{~N})$ and $120 \mathrm{~mm} / \mathrm{s}(V=-2 \mathrm{~mm} / \mathrm{s})$. The contact conditions are much severer than both the imposed conditions and the contact conditions obtained in the case presented in Section 3.5.4.1.

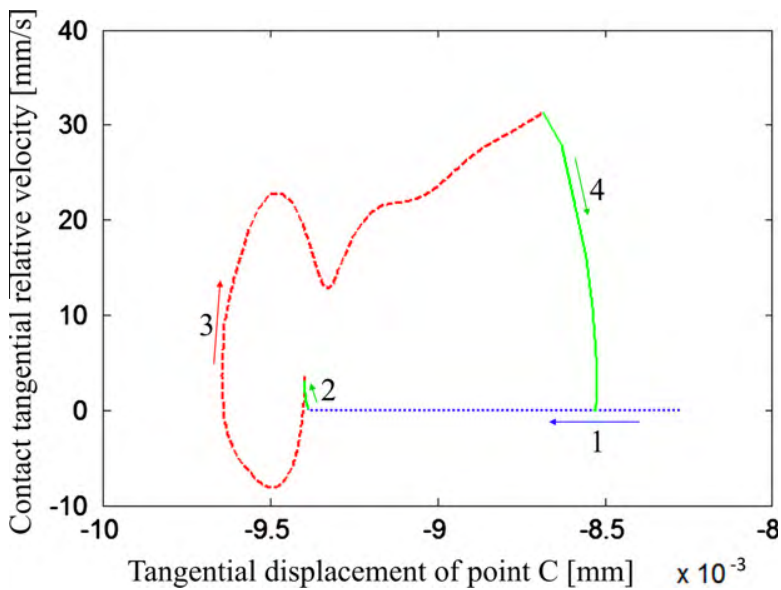

Fig. 9. Contact tangential relative velocity versus tangential displacement of node $C$ for a period of vibration. The contact slave node is successively in contact states of (1) contact stick (.....), (2) contact slip (-), (3) contact separation (- - -), and (4) contact slip $(-)$ during one period of vibration. (Numerical time domain simulation with $\mu=0.2, V=-5 \mathrm{~mm} / \mathrm{s}, F=9 \mathrm{~N}$ and $\theta=5^{\circ}$.) (For interpretation of the references to colour in this figure legend, the reader is referred to the web version of this article.) 

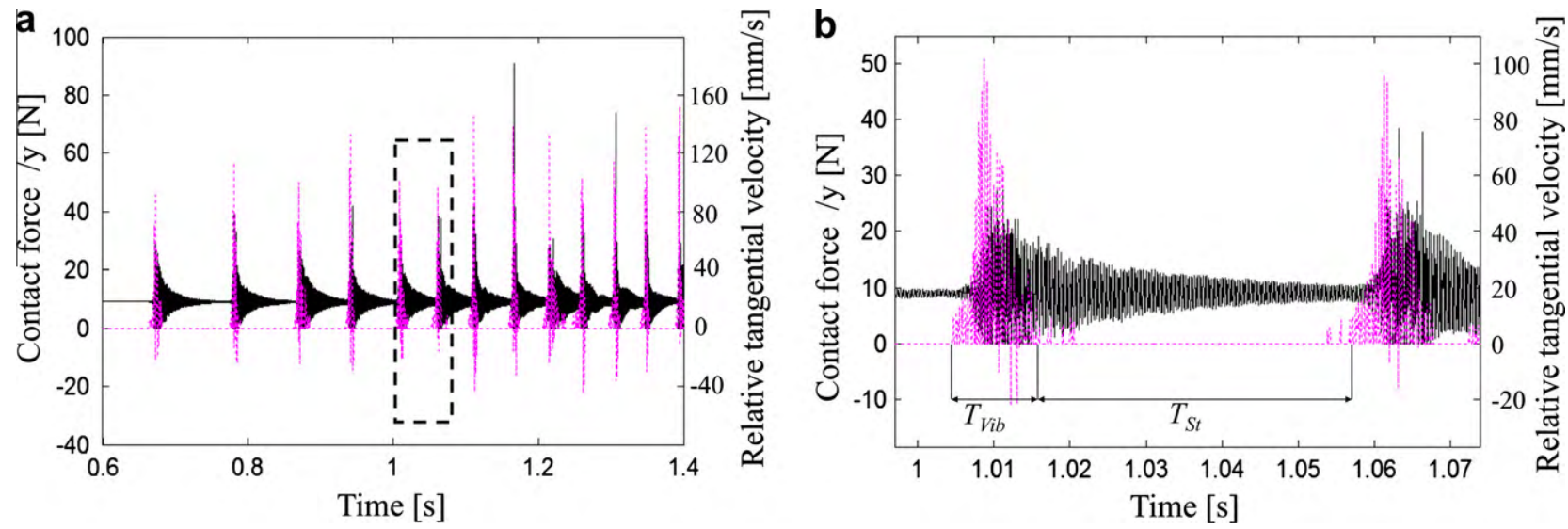

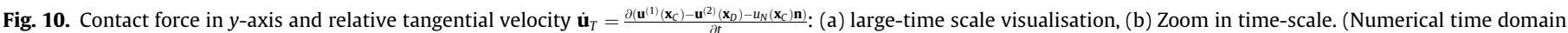
simulation with $\mu=0.2, V=-2 \mathrm{~mm} / \mathrm{s}, F=9 \mathrm{~N}$, and $\theta=5^{\circ}$.)

Then during $T_{S t}$ is the magnitude of high-frequency vibration decreases, whereas the slave node sticks to the surface of beam $\mathrm{P}^{(2)}$ (Fig. 10b).

As already observed experimentally the average duration of these sticking phases $\left(T_{S t}\right)$ is inversely proportional to the relative imposed velocity $V$ between the two beams:

$\frac{\left(T_{S t}\right)_{V=-2} \mathrm{~mm} / \mathrm{s}}{\left(T_{S t}\right)_{V=-1} \mathrm{~mm} / \mathrm{s}} \approx \frac{-1 \mathrm{~mm} / \mathrm{s}}{-2 \mathrm{~mm} / \mathrm{s}}$

Thus the translation velocity imposed on $\mathrm{P}^{(2)}$ therefore determines the type of vibrations, changing from a intermittent periodic steady state vibrating phenomenon $(|V|<5 \mathrm{~mm} / \mathrm{s})$ to a periodic steady state vibration phenomenon $(|V| \geqslant 5 \mathrm{~mm} / \mathrm{s})$. Likewise, time domain simulations (not presented here) with a velocity $V$ of $-2 \mathrm{~mm} / \mathrm{s}$ and a lower friction coefficient or a lower imposed force indicate that the system vibrated without intermittence. Reducing the friction coefficient or imposed force $F$ causes the time $T_{S t}$ that beam $\mathrm{P}^{(1)}$ sticks to beam $\mathrm{P}^{(2)}$ to decrease.
This kind of vibration can be associated to the stick-slip phenomenon [6]. Mills highlights this phenomenon for a friction coefficient that decreases with relative velocity. In our configuration, the imposed friction coefficient is constant and the vibrations correspond to a limit cycle including stick-slip-separation in the contact zone.

As highlighted here, the imposed velocity influenced the dynamic behaviour of the system during friction-induced vibrations. The variation of the normal contact force produced changes in the sliding friction force that in turn produces a tangential oscillation. Depending on the velocity $V$, this oscillation could lead to stick for short intervals of time. Moreover, with the increased in magnitude of the normal oscillations, normal separations may occur.

It has been showed that complex eigenvalue and transient dynamic analyses (performed in parallel) could predict the level and type of vibration frequency with accuracy and they explain the physical phenomena involving in friction-induced vibrations such as contact dynamics and the importance of coupling between tangential and normal vibrations at the contact.

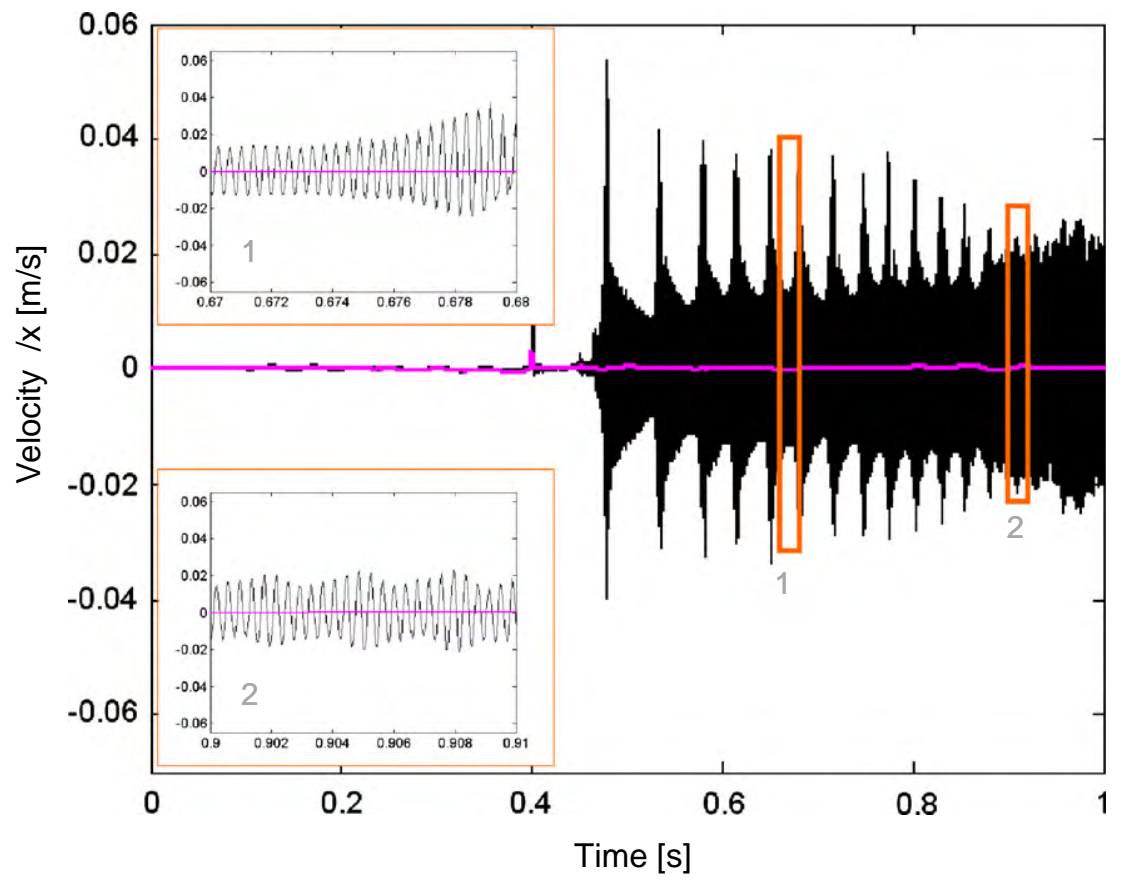

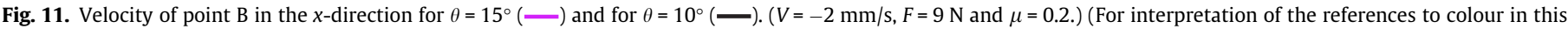
figure legend, the reader is referred to the web version of this article.) 
Table 6

Numerical results of the complex eigenvalue and dynamic transient analyses and experimental results obtained for different values of angle $\theta$. $(F=9 \mathrm{~N}$ and $\mu=0.2) \cdot \square$ : flutter instability frequencies for CEA and fundamental frequencies of vibrations spectrum for experimental an dynamic transient analysis.

\begin{tabular}{llll}
\hline Angle 0 & Linear analysis & Non-linear analysis & Experimental results \\
\hline$-15^{\circ}$ & STABLE & & \\
$-10^{\circ}$ & & & \\
$-5^{\circ}$ & & \\
$0^{\circ}$ & & \\
$5^{\circ}$ & $400 \mathrm{~Hz} 2800 \mathrm{~Hz}$ & $2700 \mathrm{~Hz}$ & \\
$10^{\circ}$ & $2800 \mathrm{~Hz}$ & & \\
$12^{\circ}$ & STABLE & \\
$15^{\circ}$ & & & \\
\hline
\end{tabular}

\subsection{Influence of angle $\theta$}

The tests were performed with different angles $\theta$. Fig. 11 shows the experimental velocity of node B in the $x$-direction for angles $\theta$ of $10^{\circ}$ and $15^{\circ}$. For an angle $\theta$ of $15^{\circ}$, the velocity tended to a null constant value and the system was in stable sliding state. For an angle $\theta$ of $10^{\circ}$, the system exhibits periodic steady state vibrations at a frequency of $2700 \mathrm{~Hz}$. The experimental results obtained for the different angles $\theta$ tested are compared to the numerical results in Table 6. For negative angles and those greater than $12^{\circ}$, the system is in stable sliding state. This results highlights that the geometry of the system and particularly the contact geometry is an important parameter for friction-induced instability. Angle $\theta$ modifies the coupling between normal and tangential degrees of freedom and thus affects the influence of friction on instability. This confirms the importance of coupling between tangential and normal vibrations in friction-induced vibrations (Section 3.4.2).

In our system the angle $\theta$ is the determining parameter of this coupling. This aspect of friction-induced vibrations has been highlighted first by Spurr [7]. For the Sprag-Slip phenomenon, the critical friction coefficient depends directly on angle $\theta$, as in the results presented here. In our system, the critical friction coefficient also depends on beam dimensions, the materials used and the position of the contact point. This dependence of inclination angle has been deeply investigated by Kang [38] in the case of a friction-induced coupled oscillator.

\section{Conclusions}

This article presented a numerical and experimental study of the friction-induced vibrations of an experimental system. Transient dynamic and complex eigenvalue analyses with a Lagrange multiplier for imposing contact constraints were performed. Their results were first correlated with experimental results with good precision with respect to frequency content and vibrations in time and then contributed to additional understanding of the physical phenomenon involved in friction-induced vibrations.

The system studied displayed friction-induced instability phenomena according to the value of the velocity of beam $\mathrm{P}^{(2)}$ and angle $\theta$. The friction-induced vibrations come from a phenomenon of flutter instability. Linear analysis showed that this instability corresponds to coalescence between two modes whose modal amplitude of displacement in the contact is significant. Their frequency corresponds to the experimental frequency of vibration during friction-induced instability. The non-linear analysis showed that when the system is unstable, the contact conditions (contact force and relative velocity of sliding) can be more severe than the imposed conditions (imposed velocity $V$ and applied force $F$ ). This knowledge of contact conditions (forces and contact state) can explain certain experimental observations of contact surfaces. Non-linear analysis provides the complete spectrum of vibrations (fundamen- tal frequency, harmonics and other frequencies) which is important for estimating the acoustical consequences of the vibrations that occur under such contact conditions.

\section{Acknowledgments}

The authors would like to thank Claude GODEAU, Yves BERTHIER and Francesco MASSI for their technical support and valuable comments.

\section{Appendix A. Construction of matrix G}

The construction of the matrix $\mathbf{G}$ is described in this paragraph. First a projection of slave node $\mathrm{C}$ on the target segments belonging to $P^{(2)}$ is proceeded to identify the target segment of $P^{(2)}$ likely to be in contact with slave node $C$. For the sake of simplicity, a null initial gap function $\left(g\left(\mathbf{x}_{C}\right)=0\right)$ is imposed and the target segments are not approximated by bicubic splines (Fig. A.1.).

$\mathbf{G}$ is the constraint matrix coupling the slave node $\mathrm{C}$ and the target nodes $\mathrm{J}$ and $\mathrm{K}$. Assuming node $\mathrm{C}$ remains in contact during deformation without sliding on segment $[\mathrm{JK}]$ and $\alpha \in[0,1]$, then:

$$
\begin{aligned}
\left\{\begin{array}{l}
u_{N}\left(\mathbf{x}_{C}\right)-g= \\
u_{T}\left(\mathbf{x}_{C}\right)=u_{T}-g=\left(\mathbf{u}^{(1)}\left(\mathbf{x}_{C}\right)-\mathbf{u}^{(2)}\left(\mathbf{x}_{D}\right)\right)^{\mathrm{T}} \cdot \mathbf{n}-g=0
\end{array}\right. \\
\qquad\left(\mathbf{u}^{(1)}\left(\mathbf{x}_{C}\right)-\mathbf{u}^{(2)}\left(\mathbf{x}_{D}\right)\right)^{\mathrm{T}} \cdot \mathbf{t}=0
\end{aligned}
$$

where $\mathbf{u}^{(L)}\left(\mathbf{x}_{W}\right)$ represent the displacement of node, with $\mathrm{W}$ belonging to beam $P^{(L)}$.From (17) and $\mathbf{G U}=\left[\begin{array}{l}\mathbf{G}_{N} \\ \mathbf{G}_{T}\end{array}\right] \mathbf{U}=\mathbf{0}$, matrix $\mathbf{G}$ $(2 \times N d o f)$ can be easily constructed $[22,35,39]$. Non-vanishing coefficients of $\mathbf{G}$ correspond to the degrees of freedom of nodes $\mathbf{C}, \mathrm{J}$, and $\mathrm{K}$. Modification of $\mathbf{G}_{T}$ with sliding is treated in [24].

\section{Appendix B. The simplified Prakash-Cliffton law}

The simplified Prakash-Cliffton [33,34] law considers a nonnull response time of the tangential force $\lambda_{T}$ at a sudden change of normal force $\left|\lambda_{N}\right|$ (Fig. B.1).

The contact conditions are given by:

$\lambda_{N}^{i} \leqslant 0$ : contact if $\lambda_{N}^{i}<0$ and separation if $\lambda_{N}^{i}=0$.

If $\lambda_{N}^{i}<0:\left|\lambda_{T}^{i}\right| \leqslant \mu\left|\lambda_{N}^{i}\right|$

- If $\left|\lambda_{T c}^{i}\right|<\mu\left|\lambda_{N}^{i}\right|$, then stick $\dot{\mathbf{u}}_{T}=\mathbf{0}$ and at $t^{i}, \lambda_{T}^{i}=\lambda_{T c}^{i}$.

$$
\text { If }\left|\lambda_{T c}^{i}\right| \geqslant \mu\left|\lambda_{N}^{i}\right| \text {, then } \begin{aligned}
\left\{\begin{array}{l}
\operatorname{slip}: \dot{\lambda}_{T}^{i}=-\frac{1}{\hat{\tau}}\left(\lambda_{T}^{i}-\kappa \mu \lambda_{N}^{i}\right) \\
\exists \gamma>0 \text { s.t. } \dot{\mathbf{u}}_{T}=-\gamma \lambda_{T}^{i} \mathbf{t}
\end{array}\right. \\
\text { with } \kappa= \begin{cases}+1 & \lambda_{T c}^{i} \geq 0 \\
-1 & \lambda_{T c}^{i}<0\end{cases}
\end{aligned}
$$

where $\dot{\mathbf{u}}_{T}$ is the tangential relative velocity of the slave nodes linked to the target surface, $\mathbf{n}$ and $\mathbf{t}$ are the normal and tangential vectors respectively that define the contact and $\mu$ is Coulomb's friction coefficient. $\hat{\tau}$ is the characteristic time which is strictly positive constant of the simplified Prakash-Clifton law, $\dot{\lambda}_{T}^{i}$ is the time derivative of the tangential contact force at time $t^{i}$. The value $\lambda_{T}^{i}$ is obtained from the discretization in time of Eq. (18). The tangential force reaches Coulomb's tangential force $\left|\lambda_{T}^{i}\right|=\mu\left|\lambda_{N}^{i}\right|$ after a sliding time $\hat{\tau}$. $\lambda_{T c}^{i}$ corresponds to the value of the tangential contact force calculated considering that the contact is in stick status. Parameter $\hat{\tau}$ has been selected numerically to ensure convergence through grid size. A low value of $\left(\hat{\tau}=1 \times 10^{-7} \mathrm{~s}\right)$ is chosen to remain close to the hypothesis proposed for the complex eigenvalue analysis (no regularization). 

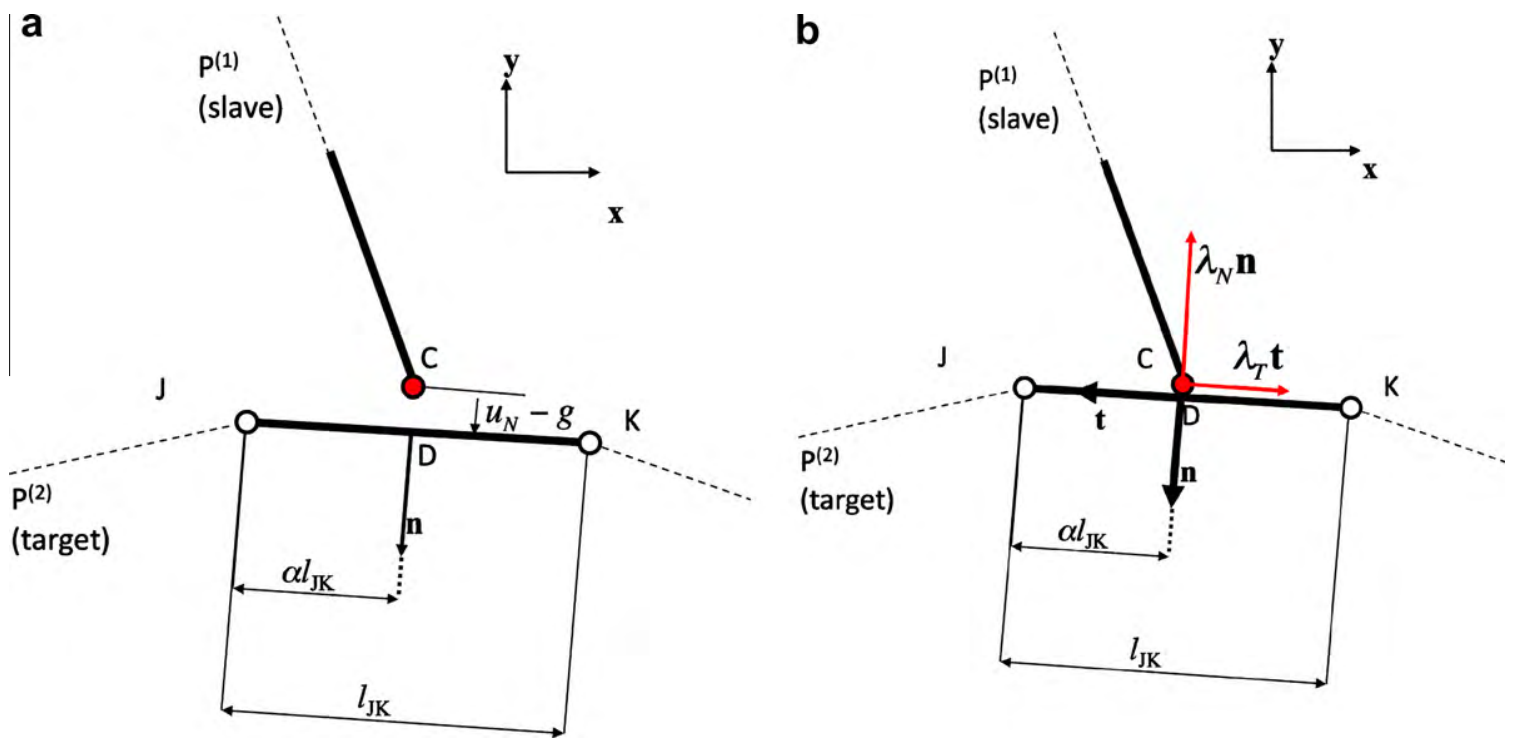

Fig. A.1. (a) Non-contact and (b) contact between $\mathrm{P}^{(1)}$ and $\mathrm{P}^{(2)}$.

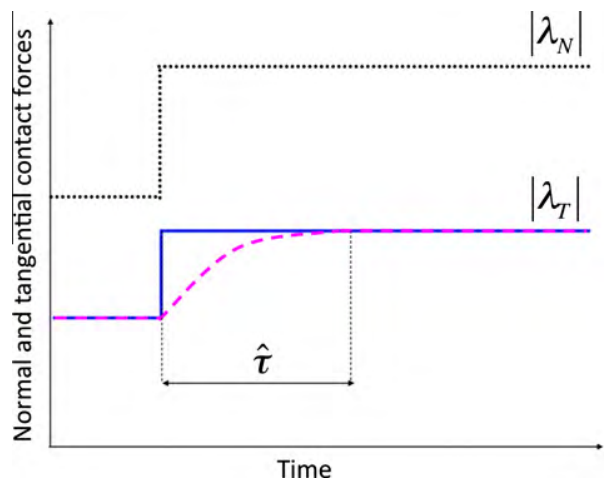

Fig. B.1. Representation of the classical Coulomb law and the simplified PrakashClifton law. The tangential force given by the classical Coulomb law (- blue) instantaneously responds a sudden variation of the normal force, contrary to that given by the simplified Prakash-Clifton law (- - , pink). (For interpretation of the references to colour in this figure legend, the reader is referred to the web version of this article.)

\section{References}

[1] Ibrahim RA. Friction-induced vibration, chatter, squeal, and chaos. Part I. Mechanics of contact and friction. ASME Appl Mech Rev 1994;47:209-26.

[2] Ibrahim RA. Friction-induced vibration, chatter, squeal, and chaos. Part II. Dynamics and modeling. ASME Appl Mech Rev 1994;47:227-53.

[3] Papinniemi A, Lai JCS, Zhao J, Loader L. Brake squeal: a literature review. Appl Acoust 2002;63(4):391-400.

[4] Kinkaid NM, O'Reilly OM, Papadopoulos P. Automotive disc brake squeal. J Sound Vib 2003;267:105-66.

[5] Akay A. Acoustics of friction. Acoust Soc Am 2002:1525-48.

[6] Mills HR. Brake squeak. Institution of Automobile Engineers; 1938

[7] Spurr RT. A theory of brake squeal. In: Proceedings of the automotive division. Institution of Mechanical Engineers; 1961. p. 33-52.

[8] North M. Disc brake squeal. Proc Inst Mech Eng 1976;C38/76:169-76.

[9] Mottershead JE. Vibration- and friction-induced instability in disks. Shock Vib Dig 1998;30(1):14-31.

[10] Liu P, Zheng H, Cai C, Wang YY, Ang KH, Liu GR. Analysis of disc brake squa using the complex eigenvalue method. Appl Acoust 2007;68(6):603-15.

[11] Tuchinda A, Hoffmann NP, Ewins DJ, Keiper W. Effect of pin finite width on instability study of the pin-on-disc system. In: Proceedings of the international modal analysis conference, vol. 1, 2002. p. 552-7.

[12] Giannini O, Sestieri A. Predictive model of squeal noise occurring on laboratory brake. J Sound Vib 2006;296:583-601.

[13] Cao Q Ouyang H, Friswell MI, Mottershead JE. Linear eigenvalue analysis of the disc-brake squeal problem. Int J Numer Methods Eng 2004;61:1546-63.

[14] Giannini O, Akay A, Massi F. Experimental analysis of brake squeal noise on a laboratory brake setup. J Sound Vib 2006;292(1-2):1-20.
[15] Baillet L, D’Errico S, Berthier Y. Influence of sliding contact local dynamics on global friction coefficient variation. Rev Eur Elém Finis 2005;14(2-3): 305-22.

[16] Oanca VG, Laursen TA. Dynamics of a state variable frictional law in finite element analysis. Finite Elem Anal Des 1996:25-40.

[17] Nguyen QS. Instability and friction. Comp Rendus Méc 2003;331(1):99-112.

[18] Chargin ML, Dunne LW, Herting DN. Nonlinear dynamics of brake squeal. Finite Elem Anal Des 1997;28:69-82.

[19] Vola D, Raous M, Martins JAC. Friction and instability of steady sliding: squeal of a rubber/glass contact. Int J Numer Methods Eng 1999;46(10):1699-720.

[20] Massi F, Baillet L, Giannini O, Sestieri A. Brake squeal: linear and nonlinear numerical approaches. Mech Syst Signal Process 2007;21(6):2374-93.

[21] Meziane A, D’Ericco S, Baillet L, Laulagnet B. Instabilities generated by friction in a pad-disc system during the braking process. Tribol Int 2007;40(7): 1127-1136.

[22] Bathe KJ. Finite element procedures in engineering analysis. NewYork: Prentice Hall; 1982. 735p.

[23] Baillet L, Sassi T. Mixed finite element formulation in large deformation frictional contact problem. Rev Eur Elém Finis 2005;14(2-3):287-304.

[24] Carpenter NJ, Taylor RL, Kantona MG. Lagrange constraints for transient finite element surface contact. Int J Numer Methods Eng 1991;32:103-28.

[25] Huseyin K. Vibration and stability of multiple parameter systems. Springer; 1978. 232p.

[26] Hetzler H. On moving continua with contacts and sliding friction: modeling, general properties and examples. Int J Solids Struct 2009;46(13):2556-70.

[27] Lorang X, Foy-Margiocchi F, Nguyen QS, Gautier PE. TGV disc brake squeal. J Sound Vib 2006;293:735-46.

[28] Martins JAC, Barbarin S, Raous M, Pino de Costa A. Dynamic stability of finite dimensional linearly elastic systems with unilateral contact and Coulomb frictio. Comput Methods Appl Mech Eng 1999;177:289-328.

[29] Tisseur F. Backward error and condition of polynomial eigenvalue problems. Linear Algebra Appl 2000;309:339-61.

[30] Massi F, Gianinni O. Effect of damping on the propensity of squeal instability: an experimental investigation. J Acoust Soc Am 2008;123(4):2017-23.

[31] Hoffmann N, Gaul L. Effect of damping on mode-coupling instability in friction-induced oscillations. ZAMM 2003;83(8):524-34.

[32] Akay A, Wickert J, Xu Z. Investigation of mode lock-in and friction interface. Final report, Pittsburgh: Department of mechanical engineering, Carnegie Mellon University; 2000.

[33] Cochard A, Rice JR. Fault rupture between dissimilar materials: illposedness, regularization and slip-pulse response. J Geophys Res 2000;105:25891.

[34] Prakash V, Clifton RJ. Time resolved dynamic friction measurements in pressure-shear. Experimental techniques in the dynamics of deformable solids, AMD-vol. 165. New-York: ASME; 1993. p. 33-48.

[35] Kikuchi N, Oden JT. Contact problems in elasticity. SIAM; 1988. 495p.

[36] Lorang X, Chiello O. Stability and transient analysis in the modelling of railway disc brake squeal, noise and vibration mitigation for rail transportation systems. Notes on numerical fluid mechanics and multidisciplinary design, vol. 99. Springer; 2008. p. 447-53.

[37] Adams GG. Steady sliding of two elastic half-space with friction reduction due to interface stick-slip. J Appl Mech 1998;65:470-5.

[38] Kang J. Parametric study on friction-induced coupled oscillator. Proc IMechE, J Mech Eng Sci 2008;222:1381-7.

[39] Belytschko $T$ et al. Non-linear finite elements for continua and structures. Wiley; 2000. 650p. 